\title{
Why oral antiseptic mouth rinsing before sputum collection cannot reduce contamination rate of mycobacterial culture in Burkina-Faso
}

\author{
Antoinette Kabore ${ }^{1}$, Juliette Tranchot-Diallo ${ }^{1,2}$, Adama Sanou ${ }^{1,2}$, Hervé Hien ${ }^{3,4}$, Géraldine Daneau \\ Michel Kireopori Gomgnimbou ${ }^{6}$, Nicolas Meda ${ }^{3,7}$, Lassana Sangaré ${ }^{7,8}$
}

1. Centre MURAZ, Department of biomedical Sciences.

2. University of Nazi Boni, UFR/ST.

3. Centre MURAZ, Department of Public Health.

4. Institut de Recherche en Science de la Santé (IRSS).

5. Institute of Tropical Medicine, Department of Biomedical Sciences.

6. University of Nazi Boni, UFR/SDS.

7. University of Pr Joseph Ki Zerbo, UFR/SDS.

8. CHU Yalgado Ouedraogo, Bacteriology-Virology Department

\begin{abstract}
Background: Tuberculosis (TB) diagnosis by culture in most resource-limited settings is hampered by high contamination rate varying up to $31 \%$. Reduction of oral microorganism loads by mouth rinse with antiseptic before sputum collection showed a reduction of contamination. Moreover, knowing the characteristic of residual contaminant microorganisms would be an asset to understand contamination issues.

Objectives: The aim of this study was to evaluate the effects of mouth rinsing with chlorhexidine on mycobacteria culture contaminations and to characterize morphologically the residual contaminants.

Methods: We consecutively included 158 patients in a TB center. Each of them supplied two sputa: The first before mouth rinse, and the second after $60 \mathrm{sec}$ of mouth rinsing with chlorhexidine $(0.1 \%)$. Petroff method and Lowenstein-Jensen media were used for sputum decontamination and inoculation respectively. The contamination rates were compared, and the type of residual contaminants were characterized and compared.

Results: The contamination rate did not differ before and after the mouth rinse (respectively 58/150 (39\%) vs $61 / 150$ (41 \%), $\mathrm{p}=0.7)$. The major residual contaminants were Gram positive spore forming bacteria $(94 \%)$.

Conclusion: Chlorhexidine mouth rinsing before sputum collection did not reduce mycobacterial culture contamination rate. This is probably due to spore forming bacteria, highlighted as major residual contaminants.

Keywords: Oral rinse, sputum, Mycobacteria culture, contamination rate, residual contaminants.

DOI: https://dx.doi.org/10.4314/ahs.v19i1.3

Cite as: Kabore A, Tranchot-Diallo J, Sanou A, Hien H, Daneau G, Gomgnimbou MK, Meda N, Sangaré L. Why Oral antiseptic mouth rinsing before sputum collection cannot reduce contamination rate of mycobacterial culture in Burkina-Faso. Afri Health Sci. 2019;19(1): 1321-1328.

bttps:/ / dx.doi.org/10.4314/abs.v19i1.3

\section{Introduction}

Tuberculosis (TB) remains a major global health problem. It causes ill-health for approximately 10 million people each year and is one of the top ten causes of death
Corresponding author:
Antoinette Kabore, Centre MURAZ, Department of biomedical Sciences Email: aswkabore@yahoo.fr

worldwide ${ }^{1}$. Although many new and fast TB diagnostic methods have been developed, culture remain the current reference standard and is still much used all around the world ${ }^{2,3}$. Unfortunately, in most resource-limited settings, diagnosis by culture is hampered by high contamination rates varying up to $31 \%$, reducing its effectiveness $s^{4,5,6,7,8,9,10}$. Recently, researchers focused on antiseptic mouth rinse before sputum collection to lower contamination ${ }^{4,10}$. Because a large size of microorganism population influences the effectiveness of antiseptics ${ }^{11}$, mouth rinse is used to reduce oral microbial load (before decon-
\end{abstract}

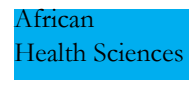

(C) 2019 Kabore et al. Licensee African Health Sciences. This is an Open Access article distributed under the terms of the Creative commons Attribution
License (https://creativecommons.org/licenses/BY/4.0), which permits unrestricted use, distribution, and reproduction in any medium, provided the original work is properly cited. 
tamination) which appear to be a potential factor linked to the contaminations. In addition, oral hygiene is insufficient particularly in Burkina-Faso, where the teeth rub sticks are the most used tool of hygiene for majority (76 $\%$ ) of individuals ${ }^{12}$. Many studies brought the proof of chlorhexidine efficiency on Gram positive and negative bacteria, as well as on fungi $1^{13,14}$, and studies on oral antiseptic rinse with chlorhexidine before sputum collection showed a reduction of contamination ${ }^{4,10}$. Nevertheless, this reduction was accompanied by a reduction on culture positivity ${ }^{4}$. Moreover, these studies did not characterize the types of residual contaminant microorganisms, which are known to have a variable sensitivity towards antiseptics $^{11}$. The aim of our study was to evaluate the impact of Chlorhexidine digluconate mouth rinsing before sputum collection on mycobacteria culture contaminations. Specifically, the contamination rate and the mycobacteria colony-forming units (CFU) score were compared before and after mouth rinse, and the types of residual contaminant microorganisms were morphologically characterized and also compared.

\section{Materials and methods Study population}

Between February 2016 and May 2017, 118 bacteriologically confirmed TB cases and 40 uninfected TB cases were consecutively recruited in the Centre Régional de Lutte Antituberculeuse (CRLAT) in Bobo-Dioulasso (Burkina-Faso). The medical officers collected socio-professional and clinical information from enrolled patients using a standardized routine questionnaire. Patients whose laboratory data were incomplete were excluded from the study.

\section{Study samples}

As per the National Tuberculosis Program (NTP) procedure, two sputa were collected in sterile containers on two consecutive days. For these two sputa supplying during the routine TB screening with Ziehl-Neelsen method, only the second sputum was used as the "Before mouth rinse" group because generally collected at the second day. An additional third sputum, was collected on the second screening day, after a mouth rinse. Mouth rinse consisted in $10 \mathrm{ml}$ of $0.1 \%$ chlorhexidine digluconate (Septyl $\mathbb{R}$ Laboratories PPM, Phnom Penh, Cambodia) for 60sec, under the supervision of the biomedical technologists, followed by an approximatively $30 \mathrm{sec}$ rinse with tap wa- ter. Collected sputum were stored at $+4^{\circ} \mathrm{C}-+8^{\circ} \mathrm{C}$ in the refrigerator and were then transported within $72 \mathrm{~h}$ on ice packs to the Bacteriology laboratory for culture processing.

To ensure the good microbiological quality of tap water, we realized three quality controls to search bacteria usually contamining water (including the aerobic spore forming bacteria). These quality controls consisted to filter the tap water with a dispositive (Sartorius) including filter membranes. After filtration, the membranes were deposited on to four type of media usually used for water analysis. After $24-48 \mathrm{~h}$ of incubation at $37^{\circ} \mathrm{C}$ or $37^{\circ} \mathrm{C}$ in anaerobiosis condition, colonies are counted and identified. During this study, the quality control was satisfactory with only two Staphylococcus sp found (0-2 bacteria for $100 \mathrm{ml}$ found; the norm is: $<10$ bacteria / $100 \mathrm{ml})$.

\section{Laboratory procedures \\ Determination of the contaminations and mycobacteria CFU score in sputum culture}

The biomedical technologists were blinded on sputum collection procedures (before or after mouth rinse). For each sample, strictly $2 \mathrm{ml}$ of sputum was decontaminated using modified Petroff method. Briefly, an equal volume of sputum sample $(2 \mathrm{ml})$ and sodium hydroxide (4 $\%$ ) were transferred into $50 \mathrm{ml}$ sterile Falcon tubes and incubated in a shaker at room temperature for $20 \mathrm{~min}$. After three series of centrifugation (3000trs / min for $20 \mathrm{~min}$ ) and resuspension with sterile distilled water, the sediment was suspended with $500 \mu \mathrm{L}$ of sterile distilled water. It was then inoculated $(0.1 \mathrm{~mL}$ in each tube), on to two classical L-J solid media and two L-J supplemented with $0.5 \%$ sodium pyruvate (Lowenstein-Jensen Medium Base, BioMaxima S.A., Velterow 5, Lublin, POLAND). Finally, the cultures were incubated at $37^{\circ} \mathrm{C}$ and were examined as previously described ${ }^{6}$. The cultures were declared contaminated only when all the four L-J media were contaminated. A repeated culture for contaminated L-J media was not done. The viability of Mycobacteria was estimated by comparing the Mycobacteria CFU score.

\section{Characterization of the types of residual contami- nants}

We characterized morphologically residual contaminant microorganisms which survived at both sodium hydroxide and the Malachite Green (Dye with antiseptic propri- 
ety composed L-J media) action and were found on the contaminated L-J media, and secondarily those that survived sodium hydroxide decontamination and were found in the decontaminated sediment. Smears realized from the sediment after sputum decontamination and from L-J contaminated media, and were stained with Gram Kit (Gram Nicole Quimica Clinica Aplicada, Spain). The residual contaminant forms and Gram-types were described by BX53 microscope (Olympus- Life Science Solutions). The suspension of colonies from L-J contaminated media at 0, 5 Mac Farland were diluted at 10-3 and inoculated on to $5 \%$ sheep-blood Columbia agar (Blood agar base, Liofilchem s.r.l Bacteriology products, Italy). Sub-cultures were incubated at $37^{\circ} \mathrm{C}$ for $24 \mathrm{~h}-48 \mathrm{~h}$ and the smear of the isolated colonies was examined after Gram staining.

\section{Quality control}

The quality control of the commercially mouthwash

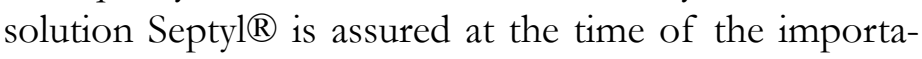
tion by the National Direction of the Pharmacy which deliver the authorization for the market of pharmaceutical products.

To prevent exogenous contaminations that can influence culture results, a sterility control of new batch of L-J media was checked macroscopically after $72 \mathrm{~h}$ of incubation at $37^{\circ} \mathrm{C}$. In addition, the laboratory participates to an external quality control from the NTP and from the National Health Laboratory Service (NHLS) in South Africa, for the Mycobacteria culture procedures.

\section{Statistical analysis}

Excel 2013 and OpenEpi 2.20 were used for data entry and analysis respectively. The contamination rate were compared using the McNemar test $(2 \times 2$ tables: focused contamination before mouth rinse vs contamination after mouth rinse) to show whether reduction of contamination is significant. The mycobacteria CFU score and the frequencies of residual contaminant microorganisms were compared using the chi-squared test or the Fischer's exact test where appropriate. Values of $\mathrm{p}<0.05$ were considered statistically significant.

\section{Ethics}

The study protocol was approved by the Institutional ethics committee. All study participants provided informed consent. The sputa specimens were coded in order to guarantee the patient confidentiality.

\section{Results}

The data analysis included 150 patients from the 158 enrolled patients. Eight (8) patients were excluded for incomplete laboratory data. Among the 150 patients, 93 (62 $\%$ ) were male: the sex ratio was 1.6. The mean age of the patients was 38 years (range: 18-88 years). Living conditions were low: $120(80 \%)$ patients lived on occasional jobs linked to poor living conditions, and 114 (76\%) were uneducated or had a primary school level.

\section{Effect of the mouth rinse with chlorhexidine on cul- ture results}

The overall contamination rate was high (40\%), and did not differ before and after the mouth rinse $(\mathrm{p}=0.7)(\mathrm{Ta}-$ ble 1).

Table 1: Effect of Chlorhexidine mouth rinse on Mycobacteria culture contamination rate

\begin{tabular}{lcccc}
\hline \multicolumn{1}{c}{ Culture results } & \multicolumn{2}{c}{ Mouth rinse } & Ch2 & $\begin{array}{c}\text { McNemar p- } \\
\text { value }\end{array}$ \\
\cline { 2 - 3 } & Before N $=150$ & After N=150 & & \\
\hline Contaminated & $61(41)$ & $58(39)$ & & \\
Not contaminated & $89(59)$ & $92(61)$ & 0.2 & \\
TB-Positive & $64(43)$ & $61(41)$ & & \\
TB- Négative & $25(17)$ & $31(21)$ & & \\
\multicolumn{2}{l}{ Note: } & & & \\
Data presented as number $(\%)$ & &
\end{tabular}


Table 2: Effect of Chlorhexidine mouth rinse on Mycobacteria colony- forming units (CFU) score

\begin{tabular}{llcccc}
\hline \multirow{2}{*}{$\begin{array}{c}\text { Microscopy } \\
\text { score }\end{array}$} & $\begin{array}{l}\text { Mycobacteria } \\
\text { CFU score }\end{array}$ & \multicolumn{2}{c}{ Mouth rinse } & Ch2 & p-value \\
\cline { 3 - 4 } & & Before N =64 & After N =61 & & \\
\hline Score $\leq 1+$ & Countable* & $4(19)$ & $5(26)$ & & \\
& Uncountable** & $17(81)$ & $14(74)$ & 0.06 & 0.8 \\
Score $>1+$ & Countable* & $4(11)$ & $8(19)$ & & \\
& Uncountable** & $39(89)$ & $34(81)$ & \multirow{2}{*}{1.6} & 0.19 \\
\multirow{3}{*}{ Total } & Countable & $8(12.5)$ & $13(21)$ & & \multirow{2}{*}{0.19} \\
& Uncountable & $56(87.5)$ & $48(79)$ & 1.7 & 0.19 \\
\hline
\end{tabular}

Notes

Data presented as number $(\%)$

NA: not applicable

* 1 to $\geq 50$ separated mycobacteria colony- forming units (CFU)

**: Numerous or very numerous, semi-confluent or confluent mycobacteria CFU

Morphological characterization and Gram type frequencies comparison of residual contaminants

On contaminated L-J media, the total number of contaminants characterized before and after mouth rinse was 97 and 96 respectively. The GPB and the bacterial spores were the major residual contaminants, with similar frequencies before and after mouth rinse for each type (GPB Around 55\%, $\mathrm{p}=0.96$; and bacterial spores 40\%, $\mathrm{p}$ $=0.87$ ) (Figure. 1). The Gram stained smear from isolated colonies showed that GPB and bacterial spores were the same microorganisms and their cumulative frequencies represented approximately $94 \%$ of the residual contaminants for both groups (Figure 1).

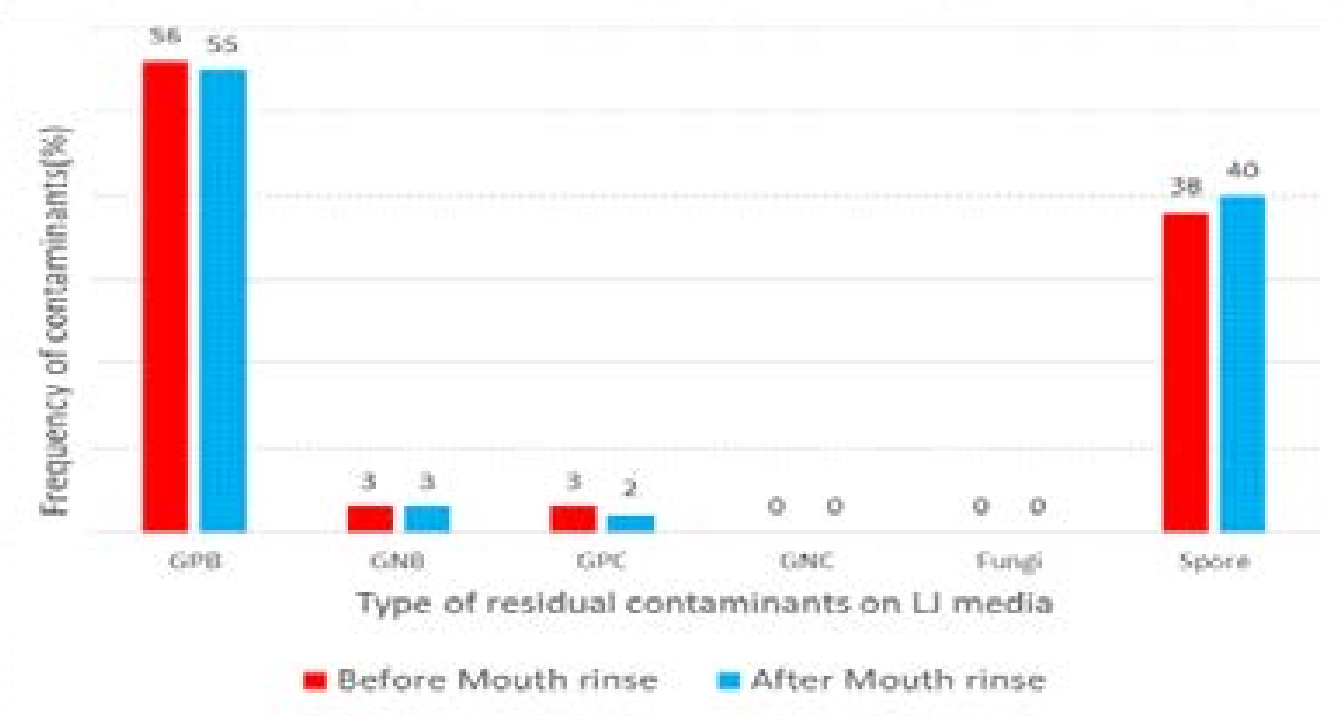

Figure 1: Frequencies of the residual contaminants from contaminated L-J media Notes: Residual contaminants on L-J: Before Mouth rinse $\mathrm{N}=97$; after Mouth rinse $\mathrm{N}=96$ ); GPB in L-J (before vs after): $p=0.96$; bacterial spores in L-J (before vs after): $p=0.87$ ). Abbreviations: GPB: Gram Positive Bacilli; GNB: Gram Negative Bacilli; GPC: Gram Positive Cocci, GNC: Gram Negative Cocci. 
Isolated bacterial spores, which could be mistaken for yeasts, were correctly identified after smear Gram staining of the isolated colonies from sub-cultures. (Image $\mathrm{C}$ and D, Fig 2). Observed endospores were warping or not the bacterial cells. Their forms were ellipsoidal or round (Figure 2).
On contaminated sediment, the total number of contaminants characterized before and after mouth rinse was respectively 100 and 75 . The GPB and fungi were the major residual contaminants. Their respective frequencies before and after mouth rinse were not statistically different respectively $(46 / 100(46 \%)$ vs $43 / 75(57 \%), p=0.3$; $23 / 100(23 \%)$ vs $14 / 75(17 \%), p=0.5)$.

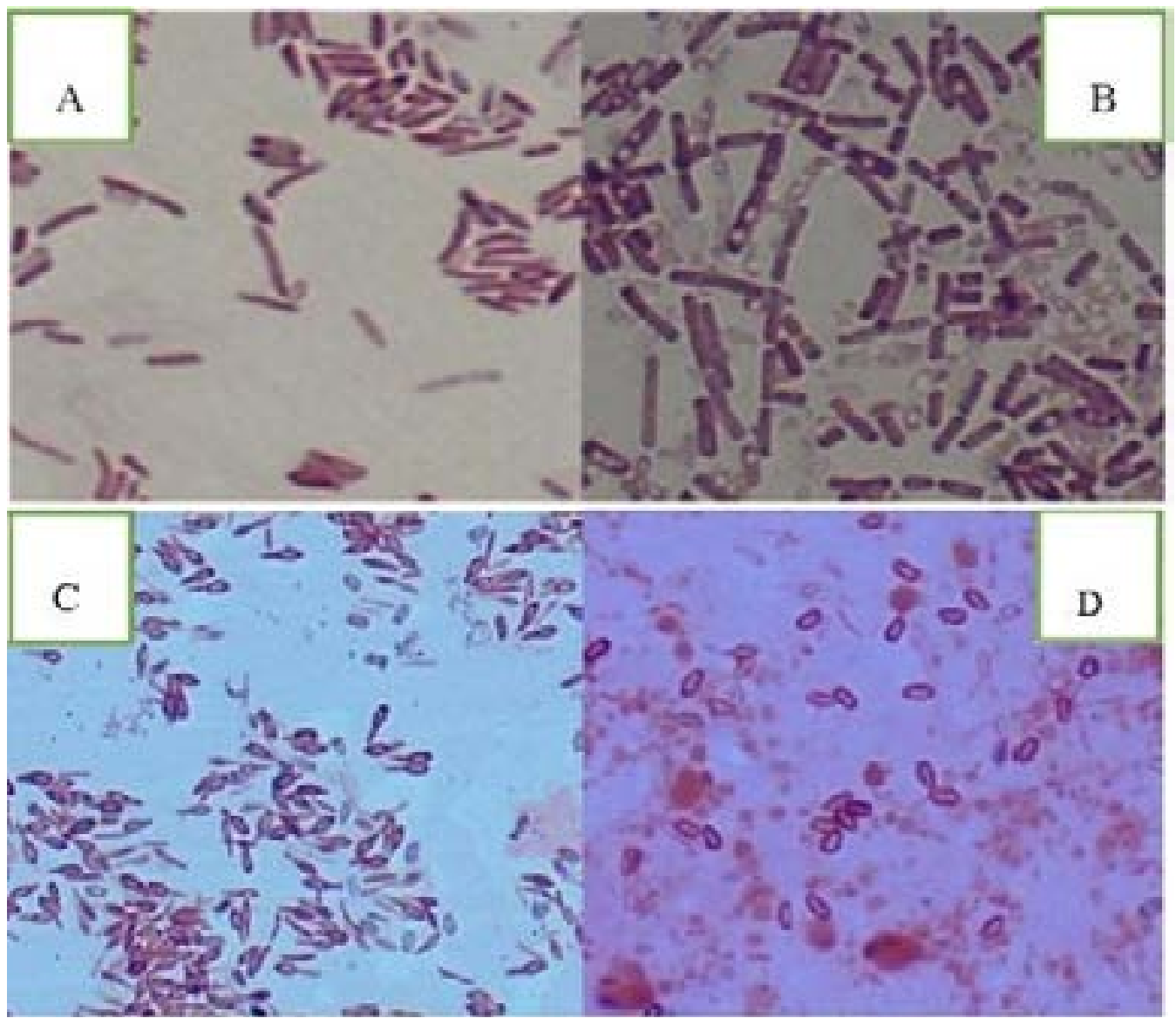

Figure 2: Microscope BX 53 image of the spore forming bacteria a major residual contaminants of mycobacteria culture (x 1000); A and B: Gram Positive Bacteria (GPB) with unwarping endospores; C and D: GPB with warping endospores. Source: Mycobacteriology laboratory, 2017.

\section{Discussion}

High contamination rate is a major barrier to Mycobacterial culture effectiveness in resource-limited settings. In this study, we evaluated the effect of the chlorhexidine antiseptic oral rinse on culture contamination rate. Our results indicate that the contaminations rates were high and were not significantly reduced by using mouth rinse. This suggests that the upstream reduction of oral microbial load does not induce downstream reduction of culture contaminations. This lack of effective action is comparable to results from Peres et al. ${ }^{10}$, in $2011(6 / 59$ $(10.2 \%)$ vs $3 / 59(5.1 \%) ; \mathrm{p}=0.38)$. However their results tended to a stronger reduction of contamination rate as compared to the findings in our study (reduction of $50 \%$ vs $4.9 \%$ ), and an initial lower contamination rate. It may partially be explained by the use of a different culture medium, the Ogawa one,known to be more selective to mycobacteria. Contrary, Kalema et al. ${ }^{4}$, in 2012 showed a significant reduction of culture contaminations after mouth rinse $(16 / 110(14.5 \%)$ vs 4/110 (3.6 \%), p 
$=0.005)$. In addition to chlorhexidine, they added nystatin as a second mouth rinse. This could have contributed to a better decontamination of the mouth. However, the types of contamination is not described by Kalema et al. ${ }^{4}$, to confirm this hypothesis. Nevertheless, as we did not highlight much contamination by fungi on L-J contaminated media, we do not expect a high impact of nystatin on our contamination rate.

In parallel to the contamination rate, our results indicate that chlorhexidine, followed by a rinse cycle with tap water, did not decrease Mycobacteria viability, including the sensitive sub-group of scanty acid fast bacilli sputa. However, as its use did not reduce culture contamination, this positive result lacks much interest.

The lack of efficiency of the mouth rinse, supposed to reduce a wide range of oral bacteria, and then to induce the reduction of contamination (after sodium hydroxide decontamination method) is not surprising in the light of our residual contaminants characterized. Indeed, we highlighted that spore forming bacteria were the major residual contaminants on L-J contaminated media, before or after mouth rinse (figure 1). This can be easier to understand because spore forming bacteria are known to be resistant to heat shock, lack of nutrients, and to most chemical substances including chlorhexidine and sodium hydroxide used for mouth rinsing and sputum decontamination in our study ${ }^{15}$. The contaminations on L-J media are observed at rather 72 hours after their inoculation that gave enough time to the spore forming bacteria to be transformed and to release bacterial spore. It is important to note that on the Gram stained smears, these bacterial forms could be easily mistaken for yeasts. The link between GPB and the spores were made by sub-cultures smears observation that clearly showed that the GPB and the bacterial spores isolated from a same contaminated L-J media represented a same micro-organism at various stages of growth. Even if Kalema et al. ${ }^{4}$, in Kampala, Uganda, and Peres et al. ${ }^{10}$ in Brazil did not characterize the residual contaminants, in our case, the spore forming bacteria highlighted as a major types of residual contaminants is probably why we observed a higher contamination rate. Because spore forming bacteria are more resistant to biocide than Mycobacteria $^{13}$, no biocide used in the current decontamination conditions can eliminate the spores while protecting the Mycobacteria. Retrospec- tively, spore forming bacteria, should also explain why numerous decontamination methods and others strategies (sputum storage conditions, mouth rinsing, adding preservative transport media to sputa etc...) to lower contamination occurrence in previous studies have not shown real satisfaction ${ }^{3,10,16,17}$.

Measures were taken to control the risk of exogenous contaminations. Besides, no contamination problem was observed when Mycobacterial strain was inoculated on L-J media during drug susceptibility testing in our laboratory. This indicator suggests an endogenous contamination of Mycobacteria culture. Although the origin of these uncommon contaminants is still unknown, they could result from the food since many studies in Africa and particularly in Burkina-Faso are usually reported that Bacillus spp (spore-forming bacteria) are the main micro-organisms responsible for fermentation of widely consumed fermented foods ${ }^{18-21}$. Biochemical and molecular characterization will be required to analyze the possible links to food contaminants, and to supply data in order to develop new strategies to lower the contamination. Beyond this study, implication of spores forming bacteria on TB-culture in resource-limited settings should be determined on a large scale and the innovative strategies of sputum decontamination should be developed in this case.

Studies of Mc Clean et al. ${ }^{16}$, in Northern Ireland, United Kingdom, and Kassaza et al. ${ }^{9}$, Mbarara, Uganda, did not realize antiseptic mouth rinse and used respectively modified Petroff method and N-acetyl-l-cysteine-sodium hydroxide method for sputum decontamination. These studies showed that the major residual contaminants were respectively Gram Negative Bacteria (GNB) 45(85 $\%)$; GPB $10(50 \%)$ and fungi two (10\%). Only one strain (2\%) of spore forming bacteria was found as residual contaminants $^{16}$. Geographical localizations, and factors specific to the host (age, race/ethnicity, environmental, health status, immunity...) appear to contribute to the variability of the oral microbiome and can explain these differences ${ }^{22,23,24}$. This suggests a specific analysis of contaminants for every laboratory confronted to contamination.

Strangely, Fungi which are regularly described to be a common contaminant in Mycobacteria culture were initially observed on contaminated sediments but not on contaminated L-J media. This domination of spore forming 
bacteria on contaminated L-J media could partially result from microorganism competition where their resistance and quickly grow allow them to take over.

\section{Conclusion}

The chlorhexidine mouth rinse before sputum collection did not reduce the contaminations rate of the Mycobacteria cultures. This is probably due to the presence of spore forming bacteria. Our results suggest that the current decontamination methods were ineffective in such situation, and better characterization of the residual contaminants are required to understand and/ or to develop innovative strategies in order to lower Mycobacterial culture contaminations, while keeping Mycobacteria viability.

Key messages: Mycobacterial culture contamination were high. Mouth rinse with chlorhexidine before sputa collection did not reduce contamination. Spore forming bacteria, for the first time highlighted as a major types of residual contaminants, which are known to be resistant to most chemical substances can explain these higher levels of contamination.

\section{Acknowledgments}

We thank the National Tuberculosis Program and the CRLAT for help in the screening and sputum collection. This study was funded by the Belgian Directorate General for Development through the Strategic Network on Tuberculosis of Institute of Tropical Medicine, Antwerp, Belgium.

\section{Competing interests}

The authors have no conflict of interest.

\section{References}

1. World Health Organization. Global tuberculosis report 2017. Available: www.who.int/entity/tb/publications/ global_report/gtbr2017_main_text.pdf.

2. Organisation Mondiale de la Santé. Tuberculose : Lignes directrices relatives à la surveillance de la pharmacorésistance. 5ème éd. WHO/HTM/TB/, 2015.

3. Jacobus HW, Jaime R. Conventional diagnostic methods. In Palomino CJ, Leão S C, Ritacco V. Tuberculosis 2007 from basic science to patient care, $1^{\text {st }}$ ed. 2007.

4. Kalema N, Boon SD, Cattamanchi A, Davis JL, Andama A, Katagira W, et al. Oral antimicrobial rinse to reduce mycobacterial culture contamination among tu- berculosis suspects in Uganda: a prospective study. PloS One. 2012; 7: e38888.

5. Redy M, Gounder S, Reid SA. Tuberculosis diagnostics in Fiji: how reliable is culture? Public Heath Action. 2014; 4: 184-188.

6. Kaboré A, Hien H, Sanou A, Zingué D, Daneau G, Ganamé Z, et al. Impact of pre-analytical factors on mycobacterium cultures contaminations rates in Burkina Faso, West Africa. Pan Afr Med J. 2014; 19:396.

7. Programme National de lutte contre la Tuberculose. Rapport annuel d'activités de lutte contre la tuberculose. Ouagadougou: PNT, 2015.

8. Ouassa TD, Zinzendorf NY, Affi MR, Menan H, Loukou GY. Evaluation des performances de trois milieux de culture des Mycobactéries à Abidjan, Côte d'Ivoire. J sci pharm Boil. 2011; 12: 50-57 PubMed .

9. Kassaza K, Orikiriza P, Llosa A, Bazira J, Dan Nyehangane D, Anne Laure P, et al. Lowenstein Jensen selective media to Reduce contamination in Tuberculosis Culture. J. Clin. Microbiol. 2014; 52(7): 2671-2673.

10. Peres RL, Palaci M, Loureiro RB, Dietze R, Johnson JL, Golub JE, et al. Evaluation of oral antiseptic rinsing before sputum collection to reduce contamination of Mycobacterial cultures. J Clin Microbiol. 2011; 49: 3058PubMed ;3060.

11. Prescott LM, Harley JP, Klein DA. Le control des microorganismes par les agents physiques et chimiques. In Microbiologie 2ème éd Française de Boeck. 2003.

12. Petersen PE, Varenne B, Ouattara S. Oral health behaviour of children and adults in urban and rural areas of Burkina Faso, Africa. International Dental Journal. 2006; 56:61-70.

13. McDonnell G, and Russell AD. Antiseptics and disinfectants: activity, action, and resistance. Clin. Microbiol. Rev. 1999; 12:147-179.

14. Grossman E, Reiter G, Styrzenberg OP. Six-month study of effects of a chlorhexidine mouth rinse on gingivitis in adults. J. Periodontal Res. 1986; 343:33-43.

15. Russell AD. Activity of biocides against Mycobacteria. Journal of Applied Bacteriology. 1996; 81: 87-101.

16. McClean M, Stanley T, Stanley S, Maeda Y, Goldsmith $\mathrm{CE}$, Shepherd R, et al. Identification and characterization of breakthrough contaminants associated with the conventional isolation of Mycobacterium tuberculosis. J Med Microbiol. 2011; 60:1292- PubMed ;1298.

17. Zingué D, Hien H, Méda N, Zida S, Kaboré A, Sanou A, et al. Advantages and drawbacks of expectoration 
decontamination methods for tuberculosis and anti-tuberculosis drug resistance diagnosis. Ann Biol Clin. 2013; 71:283-291 PubMed .

18. Savadogo A, Ilboudo AJ, Gnankine O, Traore AS. Numeration and Identification of thermo-tolerant endospore-forming Bacillus from two fermented condiments Bikalga and Soumbala. Adv. Environ. Biol. 2011; 5(9):29602966.

19. Ouoba LII, Diawara B, Jespersen L, Jakobsen M. Antimicrobial activity of Bacillus subtilis and Bacillus pumilus during the fermentation of African locust bean (Parkia biglobosa) for Soumbala production. J. Appl. Microbiol. 2007; 102:963-970 PubMed .

20. Parkouda C, Diawara B, Ouoba LII. Technology and physico-chemical characteristics of Bikalga, alkaline fermented seeds of Hibiscus sabdariffa. Afr. J Biotechnol. 2008; 7: 916-922.
21. Savadogo A Tapsoba F, Zongo C, Taale ES, Tarnagda B, Baba ML. Biofilm producing strains from local seeds foods (Zamne, Bikalga and Soumbala): Effect of glucose and agar concentrations on the biofilm production. IJAMBR. 2016; 4: 48-59 PubMed .

22. Moon JH, Lee JH. Probing the diversity of healthy oral microbiome with Bioinformatics approaches. $B M B$ Rep. 2016; 49 (12): 662-670.

23. Li1 J, Quinque D, Horz HP, Li M, Rzhetskaya M, Raff JA, et al. Comparative analysis of the human saliva microbiome from different climate zones: Alaska, Germany, and Africa. BMC Microbiology. 2014; 14:316-328.

24. Belstrom D, Fiehn NE, Nielsen CH, Kirkby N, Twetman S, Klepac-Ceraj V, et al. Differences in bacterial saliva profile between periodontitis patients and a control cohort. J Clin Periodontol. 2014; 41 (2): 104_112. 